CERN-TH/97-333

LPTHE-ORSAY 97/xx

Saclay T97/xx

hep-ph/9711517

\title{
Quark flavour conserving violations of the lepton number
}

\author{
P. Binétruy ${ }^{a, b}$, E. Dudas ${ }^{a, b}$, S. Lavignac ${ }^{a, c}$ 円 and C.A. Savoy ${ }^{d, b}$ \\ ${ }^{a}$ Laboratoire de Physique Théorique et Hautes Energies 2 \\ Bât. 210, Univ. Paris-Sud, F-91405 Orsay Cedex, France \\ ${ }^{b}$ CERN-TH, CH-1211 Geneva 23, Switzerland \\ ${ }^{c}$ Institute for Fundamental Theory, Dept. of Physics \\ Univ. of Florida, Gainesville FL 32611, USA \\ ${ }^{d}$ CEA-SACLAY, Service de Physique Théorique, \\ F-91191 Gif-sur-Yvette Cedex FranCE
}

\begin{abstract}
We study supersymmetric models of lepton and baryon number violation based on an abelian family gauge group. Due to possible lepton-Higgs mixing, the lepton violating couplings are related to the Yukawa couplings and may be generated by them even if they were absent in the original theory. Such terms may be dominant and are not given by the naive family charge counting rules. This enhancement mechanism can provide an alignment between lepton-number violating terms and Yukawa couplings: as a result they conserve quark flavour. A natural way of suppressing baryon number violation in this class of models is also proposed.
\end{abstract}

November 1997

\footnotetext{
${ }^{1}$ ATER at Université Paris 7

${ }^{2}$ Laboratoire associé au CNRS-URA-D0063.
} 
Most of the phenomenological discussions on R-parity violations in the supersymmetric extensions of the Standard Model assume that there is a single R-parity violating coupling, at least to leading order. This is often presented in parallel with the situation among the Yukawa couplings where the top Yukawa coupling is clearly leading. But if this argument has any truth in it, any theory of fermion masses should account for the relative size of R-parity violating couplings as well. In this paper, we will assume the existence of an abelian family symmetry which explains the observed hierarchies and mixings in the quark and charged lepton sectors and discuss

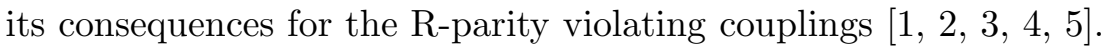

It is well-known that the simultaneous presence among $\mathrm{R}$-parity violating couplings of unsuppressed couplings violating lepton number as well as baryon number leads to dangerously fast proton decay. We will in most of what follows assume the existence of a discrete symmetry such as a baryonic parity which ensures baryon number conservation: the only allowed couplings violate lepton number. We will however relax in the end this assumption and show that family symmetries may yield a natural suppression mechanism for such couplings.

In the general approach using an abelian family symmetry to constrain the order of magnitude of Yukawa couplings [6], one describes the breaking of the family symmetry by the small parameter $\epsilon \equiv\langle\theta\rangle / M_{F}$ where $\theta$ is a field of family charge normalized to -1 and $M_{F}$ a typical flavor symmetry scale. For example denoting by $\phi_{i}$ the family charge of the superfield $\hat{\Phi}^{i}$, the coupling $\hat{\Phi}^{i} \hat{\Phi}^{j} \hat{\Phi}^{k}$ is not allowed by the family symmetry if $x_{i j k} \equiv \phi_{i}+\phi_{j}+\phi_{k} \neq 0$, but $\hat{\Phi}^{i} \hat{\Phi}^{j} \hat{\Phi}^{k} \theta^{x_{i j k}}$ is. Thus, once the family symmetry is spontaneously broken by $\langle\theta\rangle \neq 0$, the superpotential may include

$$
W \ni \lambda_{i j k} \hat{\Phi}^{i} \hat{\Phi}^{j} \hat{\Phi}^{k}
$$

with

$$
\lambda_{i j k} \sim \epsilon^{\phi_{i}+\phi_{j}+\phi_{k}} .
$$

This sort of naive power counting is actually not exact if, for some reason, the low energy fields, which we will denote by $\Phi_{i}$ do not coincide with the fields $\hat{\Phi}^{i}$ of definite family charges $\phi_{i}$. There is a possible enhancement of the low energy couplings with respect to the naive estimate (2). A standard example occurs precisely in the case of R-parity breaking: the weak doublets of hypercharge -1 and given family charge may not coincide with the Higgs doublet $H_{d}$ and the lepton doublets $L_{i}$ of the Standard Model. If they do not correspond exactly, the rotation to the low energy states -which 
are not eigenstates of the family symmetry- may yield a different order of magnitude for the low energy couplings (2) in the case where the field redefinition is nonholomorphic. We will illustrate this enhancement mechanism precisely on the R-parity violating couplings and show that it is accompanied with an alignment of these couplings along the direction of diagonal Yukawa couplings.

To be more precise, let us first write the superpotential in terms of the fields which have definite charges under the abelian family symmetry: besides the standard low energy quark and lepton superfields $Q^{k}, U^{k}, D^{k}$ and $E^{k}$, the Higgs doublet $H_{u}$ and the Standard Model singlets $\bar{N}^{k}$ of respective family charges $q_{k}, u_{k}, d_{k}, e_{k}, h_{u}, n_{k}(k=1,2,3)$, we introduce four lefthanded doublets $\hat{L}^{\alpha}$ of hypercharge -1 and family charge $l_{\alpha}(\alpha=1,2,3,4)$.

The superpotential reads:

$$
\begin{aligned}
W= & \mu_{\alpha} \hat{L}^{\alpha} H_{u}+M_{i j} \bar{N}^{i} \bar{N}^{j} \\
& +\lambda_{\alpha \beta k}^{E} \hat{L}^{\alpha} \hat{L}^{\beta} E^{k}+\lambda_{\alpha j k}^{D} \hat{L}^{\alpha} Q^{j} D^{k}+\lambda_{\alpha k}^{N} \hat{L}^{\alpha} H_{u} \bar{N}^{k}+\lambda_{i k}^{U} Q^{i} H_{u} U^{k} \\
& +\lambda_{i j k}^{\prime \prime} U^{i} D^{j} D^{k}
\end{aligned}
$$

with $\lambda_{\alpha \beta k}^{E}=-\lambda_{\beta \alpha k}^{E}$ and $\lambda_{i j k}^{\prime \prime}=-\lambda_{i k j}^{\prime \prime}$.

The standard Higgs superfield $H_{d}$ of hypercharge -1 is defined as the combination of $\hat{L}^{\alpha}$ along which $U(1)_{Y}$ is broken.

More precisely, introducing $v^{\alpha} \equiv<\hat{L}^{\alpha 0}>$,

$$
H_{d} \equiv \frac{1}{v_{d}} \sum_{\alpha} v_{\alpha} \hat{L}^{\alpha}
$$

where $v_{d} \equiv\left(\sum_{\alpha} v_{\alpha} v^{\alpha}\right)^{1 / 2}$.

The other interesting direction is along $\boldsymbol{\mu}=\left[\mu_{\alpha}\right]$ (if it is not completely aligned with $\mathbf{v}=\left[v_{\alpha}\right]$, as is generally the case). Defining therefore $\mathbf{w}=\left[w_{\alpha}\right]$ as the normed vector orthogonal to $\mathbf{v}$ in the $(\mathbf{v}, \boldsymbol{\mu})$ plane, one introduces the lepton superfield:

$$
L^{3} \equiv \sum_{\alpha} w_{\alpha} \hat{L}^{\alpha}
$$

As we will see the corresponding neutrino acquires a mass through the $\hat{L}^{\alpha} H_{u}$ terms in (3).

We can then write

$$
\hat{L}^{\alpha}=\frac{H_{d}}{v_{d}} v^{\alpha}+L^{3} w^{\alpha}+L_{\perp}^{\alpha}
$$




$$
=\frac{H_{d}}{v_{d}} v^{\alpha}+e^{\alpha}{ }_{i} L^{i}
$$

where $L_{\perp}^{\alpha}=\sum_{a=1,2} e^{\alpha}{ }_{a} L^{a}$ is orthogonal to $\mathbf{v}$ and $\mathbf{w}$ and $e^{\alpha}{ }_{3}=w^{\alpha}$.

We also introduce the angle $\xi$ between $\mathbf{w}$ and $\mathbf{v}$, i.e.

$$
\mu_{\alpha}=\mu \cos \xi \frac{v_{\alpha}}{v_{d}}+\mu \sin \xi w_{\alpha},
$$

where $\mu \equiv\left(\sum_{\alpha} \mu_{\alpha} \mu^{\alpha}\right)^{1 / 2}$.

The superpotential in (3) now reads:

$$
\begin{aligned}
W= & \mu \cos \xi H_{d} H_{u}+\mu \sin \xi L^{3} H_{u}+M_{i j} \bar{N}^{i} \bar{N}^{j} \\
& +\lambda_{i k}^{E} L^{i} H_{d} E^{k}+\lambda_{i k}^{D} Q^{i} H_{d} D^{k}+\lambda_{i k}^{N} L^{i} H_{u} \bar{N}^{k}+\lambda_{i k}^{U} Q^{i} H_{u} U^{k} \\
& +\lambda_{i j k} L^{i} L^{j} E^{k}+\lambda_{i j k}^{\prime} L^{i} Q^{j} D^{k}+\lambda_{k}^{N} H_{d} H_{u} \bar{N}^{k}+\lambda_{i j k}^{\prime \prime} U^{i} D^{j} D^{k},
\end{aligned}
$$

where

$$
\begin{array}{r}
\lambda_{i k}^{E} \equiv 2 \lambda_{\alpha \beta k}^{E} e^{\alpha}{ }_{i} \frac{v^{\beta}}{v_{d}}, \lambda_{i k}^{D} \equiv-\lambda_{\alpha i k}^{D} \frac{v^{\alpha}}{v_{d}}, \\
\lambda_{k}^{N} \equiv \lambda_{\alpha k}^{N} \frac{v^{\alpha}}{v_{d}}, \lambda_{i j k} \equiv \lambda_{\alpha \beta k}^{E} e^{\alpha}{ }_{i} e^{\beta}{ }_{j}, \\
\lambda_{i j k}^{\prime} \equiv \lambda_{\alpha j k}^{D} e^{\alpha}{ }_{i}, \lambda_{i k}^{N} \equiv \lambda_{\alpha k}^{N} e^{\alpha}{ }_{i} .
\end{array}
$$

An obvious remark at this point, which will prove to be useful in what follows, is that the baryon number violating couplings $\lambda_{i j k}^{\prime \prime}$ are not touched by the field redefinition and therefore stay independent of the Yukawa couplings. For the time being, we will set them to zero by imposing for example a baryonic parity $]^{3}$.

In the new basis $\left(H_{d}, L^{i}\right)$, the two neutrinos corresponding to $L^{a}, a=1,2$ decouple from the other states and we are left with a five-by-five neutralinoneutrino mass matrix which reads in the $\left(\tilde{\gamma}, \tilde{Z}, \tilde{H}_{u}^{0}, \tilde{H}_{d}^{0}, L_{3}^{0}\right)$ basis:

$$
M_{\nu}=\left(\begin{array}{ccccc}
M_{1} c_{w}^{2}+M_{2} s_{w}^{2} & \left(M_{2}-M_{1}\right) s_{w} c_{w} & 0 & 0 & 0 \\
\left(M_{2}-M_{1}\right) s_{w} c_{w} & M_{1} s_{w}^{2}+M_{2} c_{w}^{2} & m_{Z} \sin \beta & -m_{Z} \cos \beta & 0 \\
0 & m_{Z} \sin \beta & 0 & -\mu \cos \xi & -\mu \sin \xi \\
0 & -m_{Z} \cos \beta & -\mu \cos \xi & 0 & 0 \\
0 & 0 & -\mu \sin \xi & 0 & 0
\end{array}\right)
$$

\footnotetext{
${ }^{3} \mathrm{~A}$ simple way to obtain a baryonic parity is by the spontaneous breaking $U(1) \rightarrow Z_{N}$, which arises if the field $\theta$ which breaks $U(1)$ has a charge $N$ normalized to the smallest charge of the theory [i].
} 
where $c_{w} \equiv \cos \theta_{w}, s_{w}=\sin \theta_{w}$ and $\tan \beta \equiv v_{u} / v_{d}$.

The non-zero eigenvalue corresponding to the neutrino reads [ 8$]$

$$
m_{\nu_{3}}=m_{0} \tan ^{2} \xi
$$

with

$$
m_{0}=\frac{m_{Z}^{2} \cos ^{2} \beta\left(M_{1} c_{w}^{2}+M_{2} s_{w}^{2}\right) \mu \cos \xi}{M_{1} M_{2} \mu \cos \xi-m_{Z}^{2} \sin 2 \beta\left(M_{1} c_{w}^{2}+M_{2} s_{w}^{2}\right)},
$$

where $M_{1}$ and $M_{2}$ are the usual $U(1)_{Y}$ and $S U(2)_{L}$ gaugino soft masses.

As is well-known [9, 10], such a neutrino mass is compatible with the experimental limits only if the angle $\xi$ is small, that is in case of approximate alignment between $\mathbf{v}$ and $\boldsymbol{\mu}$. We will consider this situation in what follows.

The family symmetry gives the order of magnitude of the couplings (3), in the basis of family symmetry eigenstates. If we assume that the quadratic terms are not present in the original superpotential and are produced from the Kähler potential through the Giudice-Masiero mechanism [11], then

$$
\mu_{\alpha} \sim \tilde{m} \epsilon^{\tilde{l}_{\alpha}}
$$

where

$$
\tilde{l}_{\alpha} \equiv\left|l_{\alpha}+h_{u}\right|,
$$

$\tilde{m}$ is a typical supersymmetry breaking mass scale and $\epsilon$ measures the breaking of the family symmetry (as usual we take it to be the sine of the Cabibbo angle). Let us denote by $\tilde{l}_{0} \equiv h_{d}$ the smallest of the $\tilde{l}_{\alpha}$ and assume that $0 \leq \tilde{l}_{0}<\tilde{l}_{i}(i=1,2,3)$. Then obviously

$$
\mu_{i} / \mu_{0} \sim \epsilon^{\tilde{l}_{i}-\tilde{l}_{0}} .
$$

The components of $\mathbf{v}$ depend on $\boldsymbol{\mu}$ as well as on the soft terms in the scalar potential,

$$
V_{\text {soft }}=(B \mu)_{\alpha} \hat{L}^{\alpha} H_{u}+m_{H}^{2} H_{u}^{+} H_{u}+m_{\alpha \beta}^{2} \hat{L}^{\alpha+} \hat{L}^{\beta}+\cdots
$$

where the order of magnitude of the parameters are as follows 1 ,

$$
(B \mu)_{\alpha} \sim \epsilon^{\tilde{l}_{\alpha}} \quad m_{H}^{2} \sim \tilde{m}^{2} \quad m_{\alpha \beta}^{2} \sim \epsilon^{\left|l_{\alpha}-l_{\beta}\right|} \tilde{m}^{2} .
$$

\footnotetext{
${ }^{4} \mathrm{~A}$ more detailed determination of the soft parameters in theories with abelian family symmetries and Giudice-Masiero mechanism is given in 12 .
} 
Since $\tilde{l}_{0}<\tilde{l}_{i}$, the scalar potential can be minimized in some obvious approximations and the $\epsilon$ dependence of the $\mathbf{v}$ components can be easily obtained,

$$
v_{0} / v_{u} \sim \epsilon^{\tilde{l}_{0}} \quad v_{i} / v_{0} \sim \epsilon^{\tilde{l}_{i}-\tilde{l}_{0}} .
$$

Thus, within our assumptions,

$$
\begin{array}{cc}
v_{d} \sim v^{0} & \mu \sim \mu_{0} \\
\hat{L}^{0} \sim H_{d} & \hat{L}^{i} \sim L_{i}
\end{array}
$$

and $\tan \beta=\left(v_{u} / v_{d}\right) \sim \epsilon^{-\tilde{l}_{0}}$.

From (15) and (18), the alignement between $\mathbf{v}$ and $\boldsymbol{\mu}$ is controled by the powers $\left(\tilde{l}_{i}-\tilde{l}_{0}\right)$, so that

$$
e^{\alpha}{ }_{i} \sim \epsilon^{\left|\tilde{l}_{\alpha}-\tilde{l}_{i}\right|}
$$

Since $\sin ^{2} \xi$ can be written as

$$
\sin ^{2} \xi=\frac{\sum_{\alpha, \beta}\left(\mu_{\alpha} v_{\beta}-\mu_{\beta} v_{\alpha}\right)^{2}}{2 \mu^{2} v_{d}^{2}}
$$

one easily obtains that

$$
\sin ^{2} \xi \sim \epsilon^{2\left(\tilde{l}_{3}-\tilde{l}_{0}\right)},
$$

where $\tilde{l}_{3}$ is defined by $0 \leq \tilde{l}_{0}<\tilde{l}_{3} \leq \tilde{l}_{a}(a=1,2)$. One thus checks that the vector $w_{\alpha}$ defined in (7) is of order $\epsilon^{\left|\tilde{l}_{\alpha}-\tilde{l}_{3}\right|}$ in agreement with $w^{\alpha}=e^{\alpha}{ }_{3}$.

Let us consider the consequences of this mixing for the R-parity violating interactions. The superpotential (3) is defined in terms of the family symmetry eigenstates. Its invariance under this symmetry implies that the couplings are proportional to powers of $\epsilon$ as given by (2) if charge $\phi_{i}+\phi_{j}+\phi_{k} \geq 0$, while its analiticity implies that they vanish if the total charge is negative. From this and the $\epsilon$ dependence of the mixings in (20), one derives relations between the R-parity violating couplings and the Yukawa couplings as defined by (9). In the case where $l_{i}<l_{0}$, the former come out larger than the latter in contradiction with experimental limits. It is possible to escape this conclusion by assuming sufficiently negative $l_{i}$ charges, so that the couplings $\lambda_{i j k}^{E}, \lambda_{i j k}^{D}, \lambda_{i k}^{N}$ in (3) vanish by analyticity?. Then, in all cases,

$$
\begin{aligned}
\lambda_{i j k} & \sim \epsilon^{\tilde{l}_{i}-\tilde{l}_{0}} \lambda_{j k}^{E}, \\
\lambda_{i j k}^{\prime} & \sim \epsilon^{\tilde{l}_{i}-\tilde{l}_{0}} \lambda_{j k}^{D}, \\
\lambda_{i j}^{N} & \sim \epsilon^{\tilde{l}_{i}-\tilde{l}_{0}} \lambda_{j}^{N} .
\end{aligned}
$$

\footnotetext{
${ }^{5}$ If the gauge singlets $N_{i}$ are included in the theory, this assumption is needed in order to avoid large neutrino masses from the seesaw mechanism (cf. below).
} 
Notice that by the naive power counting the $\epsilon$ factor in (23) would be $\epsilon^{l_{i}-l_{0}}$. Therefore, according to the values of the charges $l_{\alpha}$, we distinguish four different patterns in the relations (23), as follows.

(I) $l_{i}+h_{u}>l_{0}+h_{u} \geq 0$.

In this case, all couplings in (3) are non-vanishing (unless there are zeroes in the corresponding fermion mass matrices) and, in the combinations given by the r.h.s.'s in (9), all terms such that $l_{\alpha} \leq l_{i}$, are comparable in magnitude. Then the naive power counting is preserved and the relations (23) are verified for any values of the indices, but the matrices $\lambda_{i}^{\prime}\left(\lambda_{i}\right)$ do not commute with the Yukawa $\lambda^{E}$ (resp. $\lambda^{D}$ ) matrix. Hence the R-parity violations are flavour changing in this case. These non-diagonal lepton number violations are required to be extremely small by FCNC processes, in particular by the limits on $\epsilon_{K}$ and $\Delta m_{K}$ ?. However, in models which account for realistic fermion mass hierarchies, it is sufficient to require $l_{i}-l_{0} \geq 3$ in order to fulfil these constraints. This suppresses as well quark flavour conserving R-parity violations.

(II) $l_{0}+h_{u} \leq 0<l_{i}+h_{u}$

This pattern of charges gives rise to an enhancement of flavour conserving] lepton number violation. Indeed, the naive power of $\epsilon$ would be different from that in (23) since, in this case, $\tilde{l}_{i}-\tilde{l}_{0}=\left(l_{i}-l_{0}\right)-2 \tilde{l}_{0}$. The R-parity violating couplings are larger by a factor $\epsilon^{-2 \tilde{l}_{0}}$. Furthermore, only the same terms with $\alpha=0$ (or $\beta=0$ ) in (9) contribute to this enhanced couplings as well as to the corresponding Yukawa matrices. For example,

$$
\lambda_{j k}^{D} \sim \lambda_{0 j k}^{D}, \lambda_{i j k}^{\prime} \sim \lambda_{0 j k}^{D} \epsilon^{\tilde{\epsilon}_{i}-\tilde{l}_{0}} \sim \epsilon^{-2 \tilde{l}_{0}} \epsilon^{l_{i}+q_{j}+d_{k}}
$$

and similarly for $\lambda_{j k}^{E}$ and $\lambda_{i j k}\left(\lambda_{k}^{N}\right.$ and $\left.\lambda_{i k}^{N}\right)$. Hence, the matrices of the couplings of the $H_{0}$ and all the $L_{i}$ 's are approximately proportional, i.e., aligned in the flavour space. The leading R-parity violations are predicted to be diagonal in the quark flavours. This can also be seen as a suppression of the flavour changing lepton number violations, which obey the naive power counting, and a more comfortable fulfilment of the experimental constraints

\footnotetext{
${ }^{6}$ For a review on these limits see, e.g., 13

${ }^{7}$ Notice that the alignment discussed here means that, for the mass eigenstates, the $\lambda_{i j k}^{\prime}$ matrix elements in (23) are non-vanishing for $j=k$, and that the six non-vanishing antisymmetric purely leptonic couplings $\lambda_{i j k}$ have $j=k$ or $i=k$. For simplicity, we also refer to the latter as flavour conserving lepton number violation.
} 
from FCNC processes. In this case, the flavour diagonal couplings $\lambda_{i}^{\prime}$ and $\lambda_{i}$ might be larger by a factor $\epsilon^{-2 \tilde{l}_{0}}$ as compared to case (I). This would result in a factor of $O\left(10^{3}\right)$ for the corresponding widths and cross-sections since, as already discussed, $\tilde{l}_{0}=1$ for intermediate values of $\tan \beta$.

(III) $l_{i}+h_{u}<l_{0}+h_{u} \leq 0$

As explained above, in this case the charges $l_{i}$ have to be sufficiently negative, so that the non-vanishing couplings in the right-hand side of (9) have $\alpha=0$ or $\beta=0$. One recovers the alignment in flavour space as in (24). The lepton number violating trilinear couplings are all driven by the mixing (6) induced by the misalignment between $\mathbf{v}$ and $\boldsymbol{\mu}$ so that they are fully aligned to the $H_{d}$ couplings. The power of $\epsilon$ in (23) is the opposite of the naive counting one and the R-parity violations can be suppressed by the choice of the charge differences, $l_{i}-l_{0}$, and are not constrained by $K \bar{K}$ mixing.

(IV) $l_{i}+h_{u}<0 \leq l_{0}+h_{u}$

If the charges $l_{i}$ are not negative enough to imply vanishing couplings by analyticity, the lepton number violating couplings obey the naive power counting while the Higgs couplings would get an enhancement factor of $\epsilon^{-2 \tilde{l}_{0}}$. There is no alignment and the R-parity violating couplings still supersede the Higgs couplings by a factor $\epsilon^{\tilde{l}_{i}-\tilde{l}_{0}}$ in spite of their enhancement. In order to satisfy the phenomenological constraints, the $l_{i}$ 's have to be more negative, and the pattern of lepton number violating couplings will be similar to case (III) above, with alignment given in (24) and the failure of naive power counting.

Therefore, in models with abelian charge assignments that satisfy the experimental requirements, the lepton number violating couplings obey the naive power counting in case (I), but not in the other three cases, where they are aligned to the fermion mass matrices in flavour space. Moreover, in case (II) this property is due to an enhancement of flavour diagonal R-parity violation!

An important consequence of lepton number violation is the generation of neutrino masses. At the tree-level, one neutrino gets a mass as given by (11) and (12). If the gauge singlets $N_{i}$ are not introduced in the theory, the other two neutrino states get their masses and mixings at the one loop level.

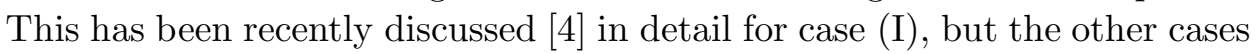
are quite similar (with the assumption made in case (III)). For completeness, we just present the general expression for the neutrino mass matrix, which 
takes into account the loop contributions as well as the seesaw masses. It can be written as follows:

$$
\left(m_{\nu}\right)_{i j}=\epsilon^{\tilde{l}_{i}+\tilde{l}_{j}-2 \tilde{l}_{0}}\left(m_{0} \delta_{i 3} \delta_{j 3}+m_{\text {loop }}+m_{\text {seesaw }}\right) .
$$

where $m_{\text {loop }}$ is a scale defined by the loop contributions [9, 14], which are dominated by the $b \tilde{b}$ one, so that [1],

$$
m_{\text {loop }} \sim \frac{1 \mathrm{keV}}{\cos ^{2} \beta}\left(\frac{500 \mathrm{GeV}}{\tilde{m}}\right),
$$

where $\tilde{m}$ is the squark mass. The seesaw contribution corresponds to the scale,

$$
m_{\text {seesaw }} \sim(1 \mathrm{eV}) \epsilon^{2\left(l_{0}+h_{u}\right)}\left(\frac{10^{13} \mathrm{GeV}}{M_{R}}\right),
$$

where we have introduced the large scale $M_{R}$ such that the $N_{i}$ mass matrix in the superpotential (3) is of the form $M_{i j} \sim \epsilon^{n_{i}+n_{j}} M_{R}$. For $M_{R}>10^{10} \mathrm{GeV}$, the loop contribution dominates over the seesaw mechanism. For the sake of comparison, we approximate (12) by

$$
m_{0} \sim(100 \mathrm{GeV}) \cos ^{2} \beta\left(\frac{500 \mathrm{GeV}}{\tilde{m}}\right),
$$

and we recall that $\cos \beta \sim \epsilon^{\tilde{l}_{0}}$. In order to satisfy the cosmological limits on the $\nu_{\tau}$ mass, we must require $\tilde{l}_{3} \geq 7$. We refer to [4] for a detailed phenomenological discussion of these predictions, which extend to the three cases above as already remarked.

It is worth noticing that the powers of $\epsilon$ in the neutrino mass matrix (25) are the same that appear in the relation between lepton number violating couplings and the Yukawa couplings in (23), providing relations for the magnitudes of these physical quantities. For example

$$
m_{\nu_{3}} \sim m_{0}\left(\frac{\lambda^{\prime}{ }_{3 j k}}{\lambda_{j k}^{D}}\right)^{2}
$$

We now come back to the problem of baryon number violation in this class of models. We already noticed that there is a qualitative difference between the couplings $\lambda^{\prime \prime}$ and $\lambda, \lambda^{\prime}$. The latter, even if for some symmetry reason they are absent from the superpotential, can be generated for leptons and down quarks through the Higgs-lepton mixing that we discussed 
previously. On the contrary, if a symmetry reason forbids the former $\left(\lambda^{\prime \prime}\right)$ couplings in the superpotential and allow them in the Kähler potential, they will only appear after supersymmetry breaking through the Giudice-Masiero mechanism [11]. More precisely, if $u_{i}+d_{j}+d_{k}<0$ for all $i, j, k=1,2,3$, then at high energy we have terms in the Kähler potential of the type $\frac{1}{M_{P}} \bar{\epsilon}^{-\left(u_{i}+d_{j}+d_{k}\right)} U^{i} D^{j} D^{k}$. Then we get contributions in the low-energy effective superpotential

$$
W \sim \frac{\tilde{m}}{M_{P}} \epsilon^{\left|u_{i}+d_{j}+d_{k}\right|} U^{i} D^{j} D^{k},
$$

where $M_{P}$ is the Planck mass. We therefore find suppressed couplings, of order $10^{-17}$ to $10^{-19}$ depending on the specific model, for moderate negative quark charges. Combining these values with the corresponding ones for $\lambda^{\prime}$ as discussed previously, we find that the proton decay can be suppressed down to acceptable values which could be tested in the forthcoming years. Of course, not all the couplings (30) are equally dangerous and this discussion can be refined in a specific model.

Because of the usual quark Yukawa couplings, this mechanism generally asks for large positive $q_{i}$ charges and is constrained by the $U(1)$ anomaly cancellation conditions. We have searched for explicit solutions in models based on a family $U(1)$ symmetry [15], [16], [18] with anomaly cancellation à la Green-Schwarz [17]. By imposing anomaly conditions and using explicit models [18], we found that the mechanism can be implemented in cases (III) and (IV) with the standard particle content. We give as an illustration one model with the following charge assignements:

$$
\begin{array}{r}
q_{1}=6, q_{2}=5, q_{3}=3, u_{1}=7, u_{2}=4, u_{3}=2, \\
d_{1}=-7, d_{2}=-8, d_{3}=-8, l_{1}=-8, l_{2}=-8, l_{3}=-8 .
\end{array}
$$

In this example, the relevant couplings mediating proton decay satisfy $\lambda^{\prime} \lambda^{\prime \prime} \leq$ $10^{-16} \epsilon^{16} \sim 10^{-26}$ for $\epsilon \simeq 0.22$, which shows the high degree of suppression which can be obtained if such couplings are obtained through the GiudiceMasiero mechanism. We emphasize that if we relax the anomaly cancellation conditions (by allowing the presence of exotic particles in the spectrum) models can be proposed with more moderate values of the charges and efficient suppression of proton decay.

\section{References}

[1] V. Ben-Hamo and Y. Nir, Phys. Lett. B339 (1994) 77. 
[2] T. Banks, Y. Grossman, E. Nardi and Y. Nir, Phys. Rev. D52 (1995) 5319.

[3] P. Binétruy, S. Lavignac and P. Ramond, Nucl. Phys. B477 (1996) 353.

[4] F. Borzumati, Y. Grossman, E. Nardi and Y. Nir, Phys. Lett. B384 (1996) 123.

[5] H.-P. Nilles and N. Polonski, Nucl. Phys. B484 (1997) 33.

[6] C. D. Froggatt and H.B. Nielsen, Nucl. Phys. B147 (1979) 277.

[7] L.E. Ibanez and G.G. Ross, Nucl. Phys. B368 (1992) 3.

[8] R. Hempfling, Nucl. Phys. B478 (1996) 3.

[9] L.J. Hall and M. Suzuki, Nucl. Phys. B231 (1984) 419

[10] L.H. Lee, Phys. Lett. 138B (1984) 121; G.G. Ross and J.W.F. Valle, Phys. Lett. 151B (1985) 375; J. Ellis et al., Phys. Lett. 150B (1985) 142; S. Dawson, Nucl. Phys. B261 (1985) 297; A. Santamaria and J.W.F. Valle, Phys. Lett. B195 (1987) 423; D.E. Brahm, L.J. Hall and S. Hsu, Phys. Rev. D42 (1990) 1860.

[11] G. Giudice and A. Masiero, Phys. Lett. B206 (1988) 480.

[12] C. Grojean, E. Dudas, S. Pokorski and C.A. Savoy, Nucl. Phys. B481 (1996) 85.

[13] H. Dreiner, to be published in "Perspectives on supersymmetry", ed. G.L. Kane, World Scientific, hep-ph/9707435.

[14] S. Dimopoulos and L.J. Hall, Phys. Lett. B207 (1987) 210; K.S. Babu and R.N. Mohapatra, Phys. Rev. Lett. 64 (1990) 1705; R. Barbieri et al., Phys. Lett. B252 (1990) 251; E. Roulet and D. Tommasini, Phys. Lett. B256 (1991) 218; K. Enqvist, A. Masiero and A. Riotto, Nucl. Phys. B373 (1992) 95; R.M. Godbole, P. Roy and X. Tata, Nucl. Phys. B401 (1993) 67; B. de Carlos and P. L. White, Phys. Rev. D55 (1997) 4222 .

[15] M. Leurer, Y. Nir and N. Seiberg, Nucl. Phys. B309 (1993) 337.

[16] L.E. Ibanez and G.G. Ross, Phys. Lett. B332 (1994) 100. 
[17] M. Green and J. Schwarz, Phys. Lett. B149 (1984) 117.

[18] P. Binétruy and P. Ramond, Phys. Lett. B350 (1995) 49;

V. Jain and R. Schrock, Phys. Lett. B352 (1995) 83;

E. Dudas, S. Pokorski and C.A. Savoy, Phys. Lett. B356 (1995) 45;

Y. Nir, Phys. Lett. B354 (1995) 337. 